\title{
Energy spectrum of cosmic rays measured using the Pierre Auger Observatory
}

\section{Vladimír Novotný ${ }^{a, *}$ on behalf of the Pierre Auger $^{b}$ Collaboration}

(a complete list of authors can be found at the end of the proceedings)

${ }^{a}$ Institute of Particle and Nuclear Physics, Faculty of Mathematics and Physics, Charles University, V Holešovičkách 2, 18000 Prague, Czech Republic

${ }^{b}$ Observatorio Pierre Auger, Av. San Martín Norte 304, 5613 Malargüe, Argentina

E-mail: spokespersons@auger.org

We present the energy spectrum of cosmic rays measured at the Pierre Auger Observatory from $6 \times 10^{15} \mathrm{eV}$ up to the most extreme energies where the accumulated exposure reaches about $80000 \mathrm{~km}^{2} \mathrm{sr}$ yr. The wide energy range is covered with five different data sets: events detected by the surface detector (with separate reconstruction methods for zenith angles below and above $60^{\circ}$ ), those collected by a denser array, a set of hybrid events simultaneously recorded by the surface and fluorescence detectors, and those events in which the signal is dominated by Cherenkov light registered by the high-elevation telescopes. In this contribution, we report updates of the analysis techniques and present the spectrum obtained by combining the five different measurements. Spectral features occurring in the wide energy range covered by the Observatory are discussed.

$37^{\text {th }}$ International Cosmic Ray Conference (ICRC 2021)

July 12 th - 23rd, 2021

Online - Berlin, Germany

\footnotetext{
${ }^{*}$ Presenter
} 


\section{Introduction}

The Pierre Auger Observatory [1] is the world's largest cosmic ray observatory. It is located in the province of Mendoza, Argentina, near the town of Malargüe. One of the primary goals of the Observatory is to measure the energy spectrum of cosmic rays.

The Observatory consists of two main parts, the array of surface detector stations (SD) that extends over $3000 \mathrm{~km}^{2}$ and the detector composed of 27 fluorescence telescopes (FD) that overlook the atmosphere above the array. These detectors operate both separately and also using a common trigger sequence in a hybrid regime. The energy spectrum of cosmic rays is measured using several detection techniques as explained in Section 2. Different techniques allow us to derive the energy spectrum in different energy ranges. The high-elevation Auger telescopes (HEAT), an extension of the FD, are used to obtain the spectrum at energies below $10^{17} \mathrm{eV}$. Between $10^{17} \mathrm{eV}$ and $10^{18.4} \mathrm{eV}$ the SD with $750 \mathrm{~m}$ spacing provides the most precise measurement while, at the highest energies, the horizontally looking FD telescopes and the SD with $1500 \mathrm{~m}$ spacing are utilized.

The energy spectrum measurements performed using the above mentioned techniques are then combined into a single estimate covering the energy range from $6 \mathrm{PeV}$ up to the highest energies. Individual measurements, the combination procedure, and spectral features observed in the combined spectrum are discussed in Section 3.

\section{Methods}

At the Pierre Auger Observatory, two fundamentally different classes of measurements utilizing two complementary detectors are used to derive the energy spectrum. The SD measures particle showers on the ground, while the FD telescopes detect fluorescence and Cherenkov light emitted by showers during their propagation through the atmosphere.

The first class relies on the SD data and divides into three separate methods as explained in Section 2.1. These measurements provide a larger exposure due to the near-100\% duty-cycle of the SD. The signals at ground measured by the SD are calibrated against the almost calorimetric estimations of the shower energy provided by the FD, allowing us to measure the spectrum with a high efficiency and with an energy scale which is largely independent of air shower simulations and of assumptions in hadronic interaction models.

The second class uses events detected by the FD. Within this class is the hybrid method utilizing SD information to assist in the reconstruction of the shower axis, and the Profile-Constrained Geometry Fit (PCGF) method applied to Cherenkov-dominated events. A disadvantage of these methods lies in the reduced FD duty cycle $(\approx 13 \%$ considering clear moonless nights $)$ and in the necessity for extensive Monte Carlo (MC) simulations to estimate the exposure to cosmic ray showers. The FD measurements benefit from a very good energy resolution and the Cherenkovdominated events allows us to lower the energy threshold to a range not yet accessible to the SD. In the future, measurements from the SD $433 \mathrm{~m}$ array [2] will extend the SD capabilities.

\subsection{Surface detector}

The SD array is composed of individual stations arranged on a triangular grid with a spacing of $1500 \mathrm{~m}$ covering an area of approximately $3000 \mathrm{~km}^{2}$. Additionally, an area of $24 \mathrm{~km}^{2}$ inside the 

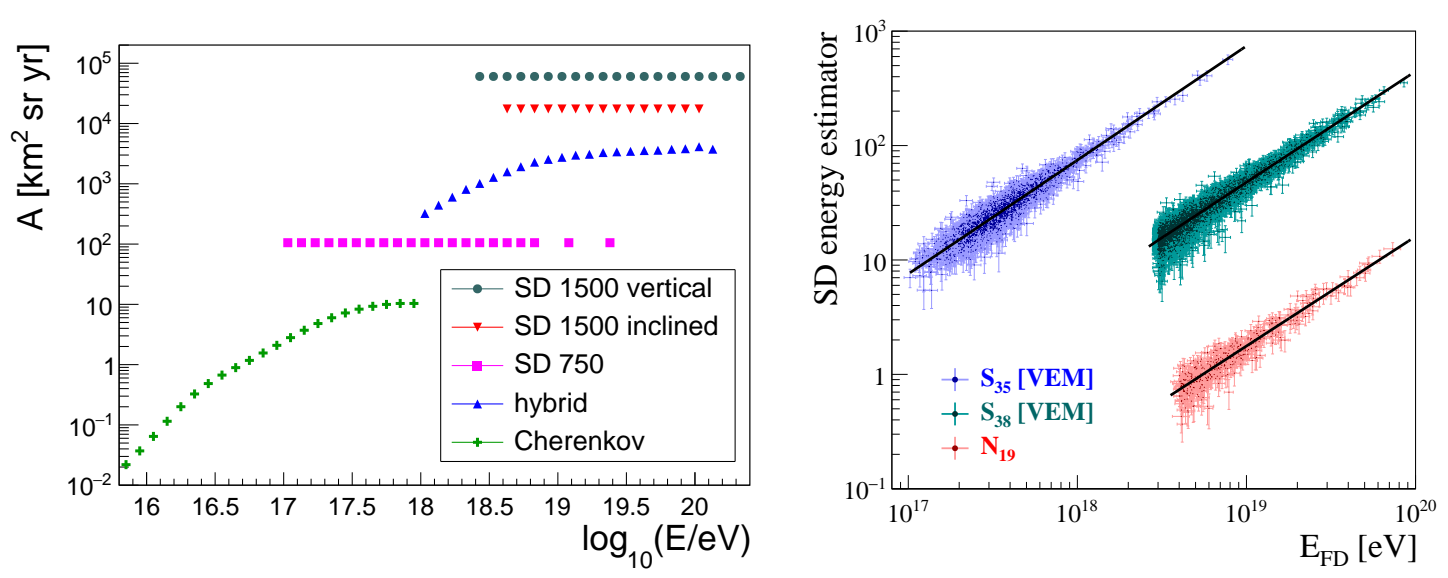

Figure 1: Exposure of SD and FD measurements to cosmic ray showers as a function of energy (left) and calibration functions of the SD energy estimators to the energies reconstructed by the FD (right).

$1500 \mathrm{~m}$ array is covered by a denser array with a spacing of $750 \mathrm{~m}$. Their spacings and areas are chosen according to the energy ranges probed by the two arrays. Individual SD stations utilize the water-Cherenkov technique of particle detection, thus they are sensitive to both the electromagnetic (EM) and muonic components of showers.

The $1500 \mathrm{~m}$ array is sensitive to cosmic ray showers with incident zenith angles up to $80^{\circ}$, but showers with zenith angles above $60^{\circ}$ (so-called "inclined" showers) are reconstructed with a different method [3] to those at lower zenith angles ("vertical" showers) [4, 5]. This is mandatory because for inclined showers the signal is dominated by muons that are deflected in the geomagnetic field producing an asymmetric footprint on the ground. For events with zenith angles below $60^{\circ}$, dominated by EM particles, this effect is negligible. The $1500 \mathrm{~m}$ array is fully efficient in the detection of showers, regardless of the primary mass composition, above $2.5 \mathrm{EeV}$ and $4 \mathrm{EeV}$ in the case of vertical and inclined reconstruction, respectively.

The array with $750 \mathrm{~m}$ spacing is designed to measure at lower energies, and is fully efficient from $0.1 \mathrm{EeV}$, assisted by an additional set of dedicated triggers [6, 7].

The aperture of all SD methods is calculated geometrically by summing the contributions from individual hexagonal cells under operation. With the use of a monitoring database, we then obtain the exposure as an integral of the aperture in time. Thus the exposure of SD measurements is independent of energy and is depicted in the left panel of Fig. 1 for all three SD methods.

The energy estimate for the $\mathrm{SD}$ array $\left(E_{\mathrm{SD}}\right)$ is obtained by means of a calibration procedure based on coincident SD and FD measurements. Events detected by both detectors can be used to obtain a relation between the SD energy estimator ( $\Sigma$ in the following) and the FD energy. This is performed using the calibration function $E_{\mathrm{FD}}=A \Sigma^{B}$, where $E_{\mathrm{FD}}$ is the energy obtained with the $\mathrm{FD}$, and $A$ and $B$ are calibration parameters.

The energy estimators in the reconstruction of vertical showers are parameters $S_{38}$ and $S_{35}$ for the SD $1500 \mathrm{~m}$ and SD $750 \mathrm{~m}$ measurements, respectively. These parameters are corrected for the average shower size attenuation in the atmosphere using the constant intensity cut method [4]. In the case of inclined reconstruction, the corresponding energy estimator is $N_{19}$, the scaling factor of the two dimensional muon density map on the ground used to fit the signal recorded by the SD [3]. The 
calibration curves are shown in the right panel of Fig. 1 and are used to estimate the energy for the bulk of SD events. The systematic uncertainty in the energy scale is 14\% [8], almost constant with energy, being dominated by the uncertainty in the absolute calibration of the FD telescopes. Finally, using the data sets that provide the energy calibration curves, we determine the ratio distributions of the SD energy to the FD energy. By fixing the FD energy resolution to $7-9 \%$ (an estimation addressed in [9]) we infer the resolution of SD energy. These estimates decrease with energy from about $20 \%$ to slightly below $10 \%$ for all the energy estimators.

\subsection{Fluorescence detector}

The FD of the Pierre Auger Observatory is composed of 27 telescopes located at four sites overlooking the SD $1500 \mathrm{~m}$ array. Each site houses 6 horizontally-looking telescopes and, additionally, 3 telescopes of HEAT are located at the Coihueco site in the vicinity of the SD $750 \mathrm{~m}$ array. Horizontally-looking telescopes cover the field of view between $0^{\circ}-30^{\circ}$ in elevation, while HEAT telescopes look higher at elevations of $30^{\circ}-60^{\circ}$ in their default mounting position.

There are two methods of air shower reconstruction used for FD measurements. The hybrid approach utilizes the time information from the SD to constrain the arrival time and impact point of the shower at the ground, which significantly improves the angular precision of the reconstruction. This method is applicable to events where the signal in the FD is dominated by fluorescence light, i.e. when showers are seen from the side. The amount of fluorescence light needed to trigger the horizontally-looking telescopes is sufficient for showers with energies above roughly $1 \mathrm{EeV}$, the threshold for the hybrid spectrum measurement.

The second approach to the shower axis determination uses the Profile-Constrained Geometry Fit (PCGF), originally developed by the HiRes collaboration [10]. This method scans over all shower geometries compatible with the arrival times of photons at individual pixels of the FD camera and for each such geometry calculates a trial energy deposit profile of the shower in the atmosphere. The geometry that provides the best expectation of the energy deposit profile from a cosmic ray shower is chosen [11]. On one hand, the precision of the PCGF reconstruction is generally lower than that obtained from the hybrid method. On the other hand, it is currently the best reconstruction method for events dominated by Cherenkov light for which the hybrid reconstruction cannot be used due to the proximity of the impact point of the shower to the position of the FD telescope. This proximity is a consequence of the beamed nature of Cherenkov light around the shower axis.

The exposure calculation of both FD reconstruction methods relies on detailed simulations of extensive air showers, light emission in the atmosphere, and the detector status described for hybrid measurements in [12]. For the Cherenkov-dominated events we utilize the same machinery except for the SD-related part. At the Pierre Auger Observatory, a detailed monitoring of the detector and atmosphere status is performed and stored for each 10 minute interval. This allows us to precisely determine the response of the FD to showers at a particular time. A re-examination of these MC simulations, which also take into account the badly calibrated telescope camera pixels that occur from time to time in the data acquisition, has led to an improved estimation of the exposure of the hybrid measurement that has resulted in a better hybrid-based estimation of the energy spectrum in comparison with the one reported in [13].

The lowest energy events accessible at the Observatory are reconstructed using the PCGF method, utilizing the fact that these showers trigger the FD with Cherenkov light which decreases 

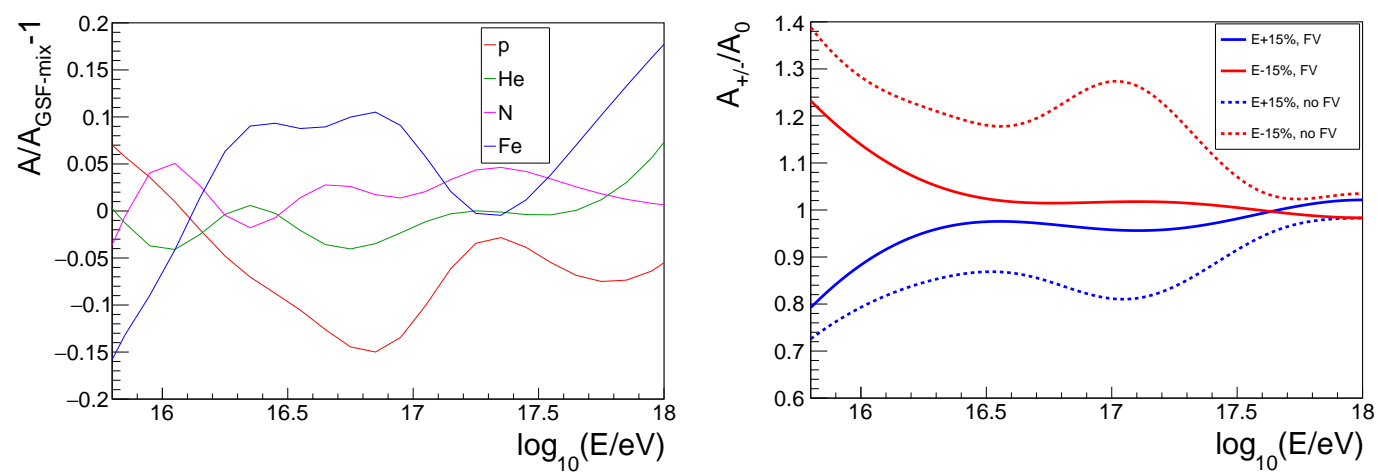

Figure 2: Systematic uncertainty of the exposure, $A$, to Cherenkov-dominated events caused by unconstrained composition of cosmic rays (left) and systematic changes in the energy assignment (right), both as a function of energy. The exposure uncertainty that would be present in the case of no FV cuts is also shown in the right panel. $A_{\mathrm{GSF}-m i x}$ signifies the exposure with the benchmark composition assumption, $A_{+/-}$ represents the exposure after the energy assignment shift, and $A_{0}$ is that assuming the nominal energy.

the energy threshold of the measurement. For this purpose we use events detected by the 9 telescopes at the Coihueco site. The FD trigger sequence was originally designed to reduce the number of Cherenkov-dominated triggers to provide the lowest possible dead time for high energy fluorescence measurements. A fraction of events that do not pass all trigger criteria is randomly stored. This fraction is $10 \%$ in the case of HEAT which results in a minimum bias data stream exploited in the calculation of the Cherenkov energy spectrum.

Changes to the reconstruction procedure described in [11] include a better description of Cherenkov light emission from showers, tuned to detailed 3D simulations in CORSIKA [14] using the CERENKOV option. We have also applied new selection cuts that helped to reduce the energy reconstruction bias to below $5 \%$ over the whole investigated energy range. The energy reconstruction resolution evolves from $12 \%$ at $10^{15.8} \mathrm{eV}$ down to $6 \%$ at $10^{18} \mathrm{eV}$. These reconstruction effects are corrected for in the forward-folding procedure of the combined fit, see Section 3. Moreover, the HEAT calibration was recently updated which improved the agreement between energy estimates from the HEAT and Coihueco telescopes.

The exposure of the PCGF measurement, as derived from realistic MC simulations, is visualized in Fig. 1. It is associated with several systematic uncertainties. The most important is the dependence of the exposure on the mass composition of cosmic rays. We use primary fractions derived from the Global Spline Fit model [15] as a benchmark assumption for the mass composition. Simulations of pure beams are then used to assess the composition uncertainty of the exposure. It accounts for up to $15 \%$ and is depicted in the left panel of Fig. 2. We have checked that the spectra calculated for zenith angles below and above $45^{\circ}$ differ by less than $\pm 10 \%$ in flux. This is compatible with the mass composition uncertainty of exposure in the corresponding zenith angle ranges.

Another uncertainty in the exposure is caused by a possible dependence of the FD trigger threshold on a systematic shift in the assignment of shower energy [12]. By changing the energy assignment we account for a potentially different number of photons emitted from the shower resulting in a different number of detected photo-electrons needed to trigger the FD telescope. This uncertainty is reduced by fiducial volume (FV) cuts that act on the shower distance to the FD telescope and the viewing angle of the shower maximum. The effect of these cuts on the exposure 
uncertainty is shown in the right panel of Fig. 2, estimated by shifting the energy assignment in MC in accordance with the $15 \%$ systematic uncertainty in the energy scale.

The energy scale uncertainty of the Cherenkov-dominated data is slightly larger than that affecting showers at higher energies [8], since it also accounts for the uncertainty in the Cherenkov emission model estimated to be $3 \%$ in energy, it includes a contribution related to the invisible energy model [11], and incorporates $2.5 \%$ in energy for half of the maximum reconstruction bias observed. The energy threshold of $6 \mathrm{PeV}$ accessible by Cherenkov-dominated events is mainly determined by the systematic uncertainty in exposure at low energies. We report data above an energy where the uncertainty in exposure matches the uncertainty attributed to the energy scale. Further details on the analysis of the Cherenkov events will be reported in a dedicated publication.

\section{The Auger spectrum and its features}

The measurements of the energy spectrum obtained with the $1500 \mathrm{~m}$ array using vertical events [5], inclined events [13], hybrid events, events detected by the $750 \mathrm{~m}$ array [6] and the FD events dominated by Cherenkov light are shown in the left panel of Fig. 3. The analysis and data set used for the hybrid events is the same as in [13] with the only exception being the improvement in the estimation of the exposure addressed in the previous section. Also, the data set for the $750 \mathrm{~m}$ array is the same as in [13], but now the analysis benefits from an improved absolute calibration of the HEAT telescopes and a reassessment of the trigger efficiency that affects the measurements around the threshold at $10^{17} \mathrm{eV}[6]$.

For the FD Cherenkov events, in comparison to our previous report [11, 13], the analysis has been improved in several aspects that have allowed us to lower the energy threshold from $3 \times 10^{16} \mathrm{eV}$ down to $6 \times 10^{15} \mathrm{eV}$, see Section 2.2. The data period was extended to 06/2012-12/2017 resulting in 123159 events selected for analysis. The energy spectrum of cosmic rays derived from the PCGF reconstruction method is depicted in the right panel of Fig. 3, together with systematic uncertainties. Besides the uncertainties in exposure we also show a major contribution from the energy scale uncertainty, both are discussed in Section 2.2.
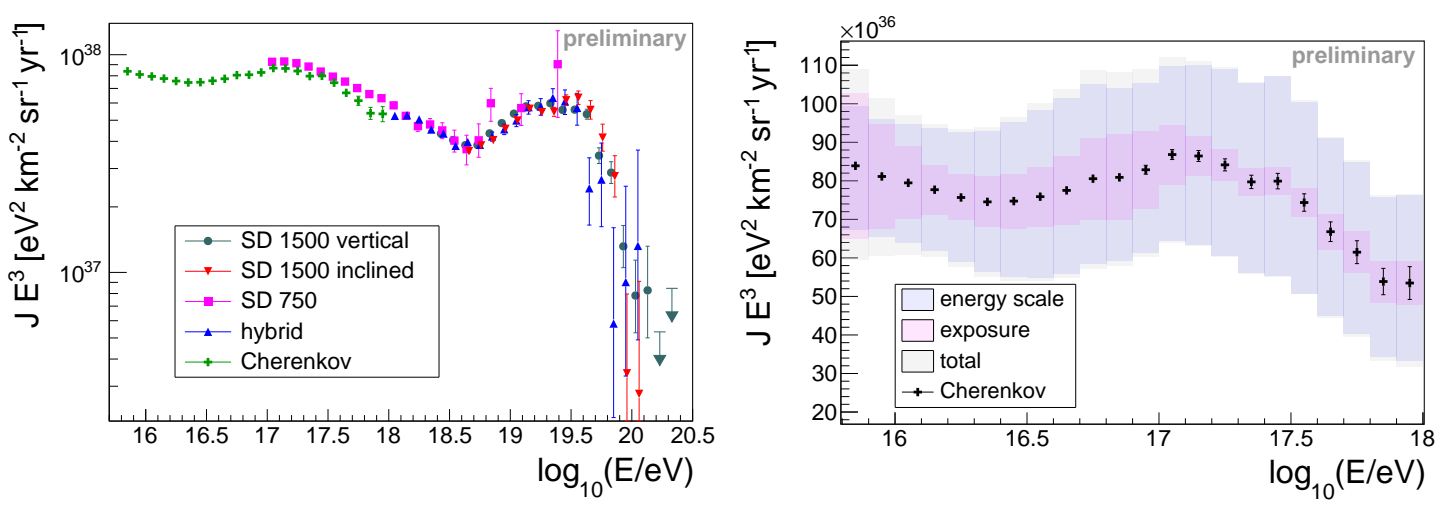

Figure 3: Intensity of cosmic rays, $J$, multiplied by $E^{3}$ estimated using five different techniques (left) and the energy spectrum deduced from Cherenkov-dominated data (right). In the right plot, the systematic uncertainty related to exposure is shown by the magenta band, that corresponding to the energy scale by the blue band, and the total systematic uncertainty by the gray band. 


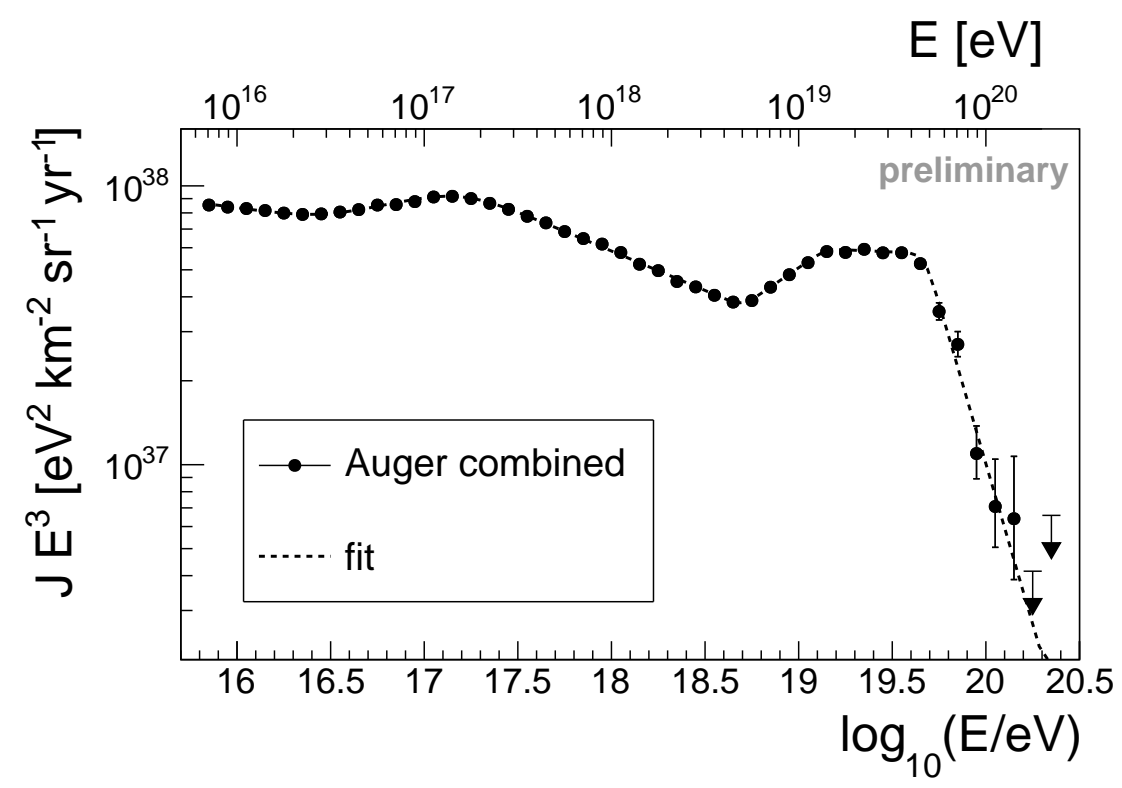

Figure 4: Combined energy spectrum multiplied by $E^{3}$ together with the fit function (dashed line).

The measurements are combined together into a single estimate of the spectrum through a forward-folding approach. First, we evaluate uncertainties uncorrelated between methods to separate them from the common uncertainty in the energy scale, the dominant uncertainty of the combined spectrum. Uncorrelated uncertainties are crucial for the combination because they serve as constraints on adjustable shifts in exposure, $\delta \mathcal{E}$, together with shifts in the energy calibration parameters of individual SD measurements, $\delta A$ and $\delta B$. Along with systematic uncertainties, we also utilize migration matrices of the individual measurement methods. These matrices describe the bin-to-bin migration of events between energy bins of true and reconstructed energy, thus taking into account energy reconstruction resolutions and biases. Using the migration matrices and measured energy distributions we apply the forward-folding procedure assuming a model for the energy spectrum that describes the data over the full energy range. This model is a function of a set of spectral parameters. Their values together with the values of $\delta \mathcal{E}, \delta A$ and $\delta B$ that best describe the numbers of detected events in all data sets are found by maximizing a combined likelihood. The likelihood is the product of the Poissonian terms, accounting for the difference between expected and measured rates per energy bin, and the Gaussian constraints on exposure and energy. The method is a generalization of the approach described in [6].

The combined spectrum and the fit function are shown in Fig. 4. The contributing spectra are found to be in agreement within their uncorrelated systematic uncertainties. At the end of the maximization, the normalization of Cherenkov and inclined spectra are shifted up by about $7 \%$ and $5 \%$, respectively. The $750 \mathrm{~m}$ array spectrum normalization is shifted down by $2 \%$ while the shifts for the other two spectra are negligible.

The model for the energy spectrum used in the forward-folding fit is a sequence of six powerlaws with spectral indexes $\gamma_{0}, \ldots, \gamma_{5}$ and five break energies $E_{01}, \ldots, E_{45}$,

$$
J(E)=J_{0}\left(\frac{E}{10^{16} \mathrm{eV}}\right)^{-\gamma_{0}} \prod_{i=0}^{4}\left[1+\left(\frac{E}{E_{i j}}\right)^{\frac{1}{\omega_{i j}}}\right]^{\left(\gamma_{i}-\gamma_{j}\right) \omega_{i j}}, \quad j=i+1,
$$


where $J_{0}$ is a normalization parameter and $\omega_{i j}$ determine the width of the transitions between the power laws.

The best-fit parameters, with statistical and systematic uncertainties, are presented in Tab. 1 . Data show with high significance the inflection points commonly called the $2^{\text {nd }}$ knee, the ankle and the abrupt suppression at the highest energies. Just above $10^{19} \mathrm{eV}$, the spectrum manifests an instep steepening point whose first observation was reported only recently [5, 16] and is now also confirmed by the Telescope Array [17]. Finally, for the first time, we report the flattening called the low energy ankle at $28 \mathrm{PeV}$ whose origin, together with that of the $2^{\text {nd }} k n e e$, is probably connected to changes in the mass composition of cosmic rays originating in our Galaxy [15, 18].

Table 1: Parameters of the best fit of Eq. (1) to the combined spectrum. The first uncertainty is statistical and the second one systematic. The fit has been performed with a set of transition width parameters that well describe the data: $\omega_{01}=\omega_{12}=0.25$ and $\omega_{23}=\omega_{34}=\omega_{45}=0.05$ [5].

$$
\begin{array}{ll|l}
\multicolumn{2}{l|}{J_{0}=(8.34 \pm 0.04 \pm 3.40) \times 10^{-11} \mathrm{~km}^{-2} \mathrm{sr}^{-1} \mathrm{yr}^{-1} \mathrm{eV}^{-1}} & \\
\text { low energy ankle } & E_{01}=(2.8 \pm 0.3 \pm 0.4) \times 10^{16} \mathrm{eV} & \gamma_{0}=3.09 \pm 0.01 \pm 0.10 \\
2^{\text {nd }} \text { knee } & E_{12}=(1.58 \pm 0.05 \pm 0.2) \times 10^{17} \mathrm{eV} & \gamma_{2}=3.283 \pm 0.001 \pm 0.05 \\
\text { ankle } & E_{23}=(5.0 \pm 0.1 \pm 0.8) \times 10^{18} \mathrm{eV} & \gamma_{3}=2.54 \pm 0.03 \pm 0.05 \\
\text { instep } & E_{34}=(1.4 \pm 0.1 \pm 0.2) \times 10^{19} \mathrm{eV} & \gamma_{4}=3.03 \pm 0.05 \pm 0.10 \\
\text { suppression } & E_{45}=(4.7 \pm 0.3 \pm 0.6) \times 10^{19} \mathrm{eV} & \gamma_{5}=5.3 \pm 0.3 \pm 0.1
\end{array}
$$

\section{References}

[1] A. Aab et al. [Pierre Auger Coll.], Nucl. Instrum. Meth. A 798 (2015) 172-213, [1502.01323].

[2] G. Silli [for the Pierre Auger Coll.], these proceedings.

[3] A. Aab et al. [Pierre Auger Coll.], JCAP 08 (2014) 019, [1407. 3214].

[4] A. Aab et al. [Pierre Auger Coll.], JINST 15 (2020) P10021, [2007.09035].

[5] A. Aab et al. [Pierre Auger Coll.], Phys. Rev. D 102 (2020) 062005, [2008.06486].

[6] A. Aab et al. [Pierre Auger Coll.], The Energy Spectrum of Cosmic Rays beyond the Turn-Down at $10^{17} \mathrm{eV}$ as Measured with the Surface Detector of the Pierre Auger Observatory, submitted to Eur. Phys. J. C.

[7] A. Coleman [for the Pierre Auger Coll.], Proc. 36th Int. Cosmic Ray Conf., Madison, USA (2019), PoS(ICRC2019)225, [1909.09073].

[8] V. Verzi [for the Pierre Auger Coll.], Proc. of 33rd Int. Cosmic Ray Conf., Rio de Janeiro, Brazil (2013), [1307. 5059].

[9] B. Dawson [for the Pierre Auger Coll.], Proc. 36th Int. Cosmic Ray Conf., Madison, USA (2019), PoS(ICRC2019)231, [1909.09073].

[10] R. U. Abbasi et al. [HiRes Coll.], Phys. Rev. Lett. 100 (2008) 101101, [astro-ph/0703099].

[11] V. Novotný [for the Pierre Auger Coll.], Proc. 36th Int. Cosmic Ray Conf., Madison, USA (2019), PoS(ICRC2019)374, [1909.09073].

[12] P. Abreu et al. [Pierre Auger Coll.], Astropart. Phys. 34 (2011) 368-381, [1010.6162].

[13] V. Verzi [for the Pierre Auger Coll.], Proc. 36th Int. Cosmic Ray Conf., Madison, USA (2019), PoS(ICRC2019)450, [1909.09073].

[14] D. Heck et al., Report (1998) FZKA-6019.

[15] H. P. Dembinski et al., Proc. 35th Int. Cosmic Ray Conf., Busan, Korea (2017), PoS(ICRC2017)533, [1711.11432].

[16] A. Aab et al. [Pierre Auger Coll.], Phys. Rev. Lett. 125 (2020) 121106, [2008. 06488].

[17] Y. Tsunesada [for the Pierre Auger and the Telescope Array Coll.], these proceedings.

[18] W. D. Apel et al. [KASCADE-Grande Coll.], Astropart. Phys. 47 (2013) 54-66, [1306.6283]. 


\section{The Pierre Auger Collaboration}

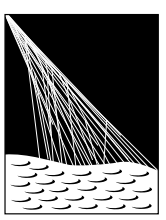

PIERRE

AUSGERVATORY

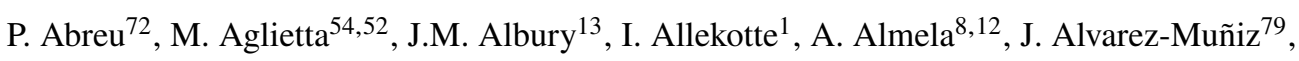
R. Alves Batista ${ }^{80}$, G.A. Anastasi ${ }^{63,52}$, L. Anchordoqui ${ }^{87}$, B. Andrada ${ }^{8}$, S. Andringa ${ }^{72}$, C. $\mathrm{Aramo}^{50}$, P.R. Araújo Ferreira ${ }^{42}$, J. C. Arteaga Velázquez ${ }^{67}$, H. Asorey $^{8}$, P. Assis ${ }^{72}$, G. Avila ${ }^{11}$, A.M. Badescu ${ }^{75}$, A. Bakalova ${ }^{32}$, A. Balaceanu ${ }^{73}$, F. Barbato ${ }^{45,46}$, R.J. Barreira Luz $^{72}$, K.H. Becker ${ }^{38}$, J.A. Bellido ${ }^{13,69}$, C. Berat ${ }^{36}$, M.E. Bertaina ${ }^{63,52}$, X. Bertou ${ }^{1}$, P.L. Biermann ${ }^{b}$, V. Binet ${ }^{6}$, K. Bismark ${ }^{39,8}$, T. Bister ${ }^{42}$, J. Biteau ${ }^{37}$, J. Blazek ${ }^{32}$, C. Bleve ${ }^{36}$, M. Boháčová ${ }^{32}$, D. Boncioli ${ }^{57,46}$, C. Bonifazi ${ }^{9,26}$, L. Bonneau Arbeletche ${ }^{21}$, N. Borodai ${ }^{70}$, A.M. Botti ${ }^{8}$, J. Brack ${ }^{d}$, T. Bretz ${ }^{42}$, P.G. Brichetto Orchera ${ }^{8}$, F.L. Briechle ${ }^{42}$, P. Buchholz ${ }^{44}$, A. Bueno ${ }^{78}$, S. Buitink ${ }^{15}$, M. Buscemi ${ }^{47}$, M. Büsken ${ }^{39,8}$, K.S. Caballero-Mora ${ }^{66}$, L. Caccianiga ${ }^{59,49}$, F. Canfora ${ }^{80,81}$, I. Caracas ${ }^{38}$, J.M. Carceller ${ }^{78}$, R. Caruso ${ }^{58,47}$, A. Castellina ${ }^{54,52}$, F. Catalani ${ }^{19}$, G. Cataldi ${ }^{48}$, L. Cazon ${ }^{72}$, M. Cerda ${ }^{10}$, J.A. Chinellato ${ }^{22}$, J. Chudoba ${ }^{32}$, L. Chytka ${ }^{33}$, R.W. Clay ${ }^{13}$, A.C. Cobos Ceruttii ${ }^{7}$, R. Colalillo ${ }^{60,50}$, A. Coleman ${ }^{93}$, M.R. Coluccia ${ }^{48}$, R. Conceição ${ }^{72}$, A. Condorelli ${ }^{45,46}$, G. Consolati ${ }^{49,55}$, F. Contreras ${ }^{11}$, F. Convenga ${ }^{56,48}$, D. Correia dos Santos $^{28}$, C.E. Covault ${ }^{85}$, S. Dasso ${ }^{5,3}$, K. Daumiller ${ }^{41}$, B.R. Dawson ${ }^{13}$, J.A. Day ${ }^{13}$, R.M. de Almeida $^{28}$, J. de Jesús ${ }^{8,41}$, S.J. de Jong ${ }^{80,81}$, G. De Mauro ${ }^{80,81}$, J.R.T. de Mello Neto ${ }^{26,27}$, I. De Mitri ${ }^{45,46}$, J. de Oliveira ${ }^{18}$, D. de Oliveira Franco ${ }^{22}$, F. de Palma ${ }^{56,48}$, V. de Souza $^{20}$, E. De Vito ${ }^{56,48}$, M. del Río ${ }^{11}$, O. Deligny ${ }^{34}$, L. Deval ${ }^{41,8}$, A. di Matteo $^{52}$, C. Dobrigkeit ${ }^{22}$, J.C. D’Olivo ${ }^{68}$, L.M. Domingues Mendes ${ }^{72}$, R.C. dos Anjos ${ }^{25}$, D. dos Santos $^{28}$, M.T. Dova ${ }^{4}$, J. Ebr ${ }^{32}$, R. Engel ${ }^{39,41}$, I. Epicoco ${ }^{56,48}$, M. Erdmann ${ }^{42}$, C.O. Escobar ${ }^{a}$, A. Etchegoyen ${ }^{8,12}$, H. Falcke ${ }^{80,82,81}$, J. Farmer ${ }^{92}$, G. Farrar ${ }^{90}$, A.C. Fauth ${ }^{22}$, N. Fazzini ${ }^{a}$, F. Feldbusch ${ }^{40}$, F. Fenu ${ }^{54,52}$,

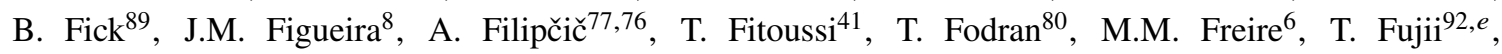
A. Fuster $^{8,12}$, C. Galea ${ }^{80}$, C. Galelli ${ }^{59,49}$, B. García ${ }^{7}$, A.L. Garcia Vegas ${ }^{42}$, H. Gemmeke ${ }^{40}$, F. Gesualdi ${ }^{8,41}$, A. Gherghel-Lascu ${ }^{73}$, P.L. Ghia ${ }^{34}$, U. Giaccari ${ }^{80}$, M. Giammarchi ${ }^{49}$, J. Glombitza ${ }^{42}$, F. Gobbi ${ }^{10}$, F. Gollan ${ }^{8}$, G. Golup ${ }^{1}$, M. Gómez Berisso ${ }^{1}$, P.F. Gómez Vitale ${ }^{11}$, J.P. Gongora ${ }^{11}$, J.M. González ${ }^{1}$, N. González ${ }^{14}$, I. Goos ${ }^{1,41}$, D. Góra ${ }^{70}$, A. Gorgi ${ }^{54,52}$, M. Gottowik ${ }^{38}$, T.D. Grubb ${ }^{13}$, F. Guarino ${ }^{60,50}$, G.P. Guedes ${ }^{23}$, E. Guido ${ }^{52,63}$, S. $\mathrm{Hahn}^{41,8}$, P. $\mathrm{Hamal}^{32}$, M.R. Hampel ${ }^{8}$, P. Hansen ${ }^{4}$, D. Harari ${ }^{1}$, V.M. Harvey ${ }^{13}$, A. Haungs ${ }^{41}$, T. Hebbeker ${ }^{42}$, D. Heck ${ }^{41}$, G.C. Hill ${ }^{13}$, C. Hojvat ${ }^{a}$, J.R. Hörandel ${ }^{80,81}$, P. Horvath ${ }^{33}$, M. Hrabovský ${ }^{33}$, T. Huege ${ }^{41,15}$, A. Insolia ${ }^{58,47}$, P.G. Isar $^{74}$, P. Janecek ${ }^{32}$, J.A. Johnsen ${ }^{86}$, J. Jurysek ${ }^{32}$, A. Kääpä ${ }^{38}$, K.H. Kampert ${ }^{38}$, N. Karastathis ${ }^{41}$, B. Keilhauer ${ }^{41}$, J. Kemp ${ }^{42}$, A. Khakurdikar ${ }^{80}$, V.V. Kizakke Covilakam $^{8,41}$, H.O. Klages ${ }^{41}$, M. Kleifges ${ }^{40}$, J. Kleinfeller ${ }^{10}$, M. Köpke ${ }^{39}$, N. Kunka ${ }^{40}$, B.L. Lago ${ }^{17}$, R.G. Lang ${ }^{20}$, N. Langner ${ }^{42}$, M.A. Leigui de Oliveira ${ }^{24}$, V. Lenok $^{41}$, A. Letessier-Selvon ${ }^{35}$, I. LhenryYvon $^{34}$, D. Lo Presti ${ }^{58,47}$, L. Lopes ${ }^{72}$, R. López ${ }^{64}$, L. Lu ${ }^{94}$, Q. Luce ${ }^{39}$, J.P. Lundquist ${ }^{76}$, A. Machado Payeras $^{22}$, G. Mancarella ${ }^{56,48}$, D. Mandat ${ }^{32}$, B.C. Manning ${ }^{13}$, J. Manshanden ${ }^{43}$, P. Mantsch ${ }^{a}$, S. Marafico ${ }^{34}$, A.G. Mariazzi ${ }^{4}$, I.C. Mariş ${ }^{14}$, G. Marsella ${ }^{61,47}$, D. Martello ${ }^{56,48}$, S. Martinelli ${ }^{41,8}$, O. Martínez Bravo ${ }^{64}$, M. Mastrodicasa ${ }^{57,46}$, H.J. Mathes ${ }^{41}$, J. Matthews ${ }^{88}$, G. Matthiae ${ }^{62,51}$, E. Mayotte ${ }^{38}$, P.O. Mazur ${ }^{a}$, G. MedinaTanco $^{68}$, D. Melo $^{8}$, A. Menshikov ${ }^{40}$, K.-D. Merenda ${ }^{86}$, S. Michal ${ }^{33}$, M.I. Micheletti ${ }^{6}$, L. Miramonti ${ }^{59,49}$, S. Mollerach ${ }^{1}$, F. Montanet ${ }^{36}$, C. Morello ${ }^{54,52}$, M. Mostafá ${ }^{91}$, A.L. Müller ${ }^{8}$, M.A. Muller ${ }^{22}$, K. Mulrey ${ }^{15}$, R. Mussa ${ }^{52}$, M. Muzio ${ }^{90}$, W.M. Namasaka ${ }^{38}$, A. Nasr-Esfahani ${ }^{38}$, L. Nellen ${ }^{68}$, M. Niculescu-Oglinzanu ${ }^{73}$, M. Niechciol ${ }^{44}$, D. Nitz ${ }^{89}$, D. Nosek ${ }^{31}$, V. Novotny ${ }^{31}$, L. Nožka ${ }^{33}$, A Nucita ${ }^{56,48}$, L.A. Núñez ${ }^{30}$, M. Palatka ${ }^{32}$, J. Pallotta ${ }^{2}$, P. Papenbreer ${ }^{38}$, G. Parente ${ }^{79}$, A. Parra ${ }^{64}$, J. Pawlowsky ${ }^{38}$, M. Pech ${ }^{32}$, F. Pedreira ${ }^{79}$, J. Pȩkala ${ }^{70}$, R. Pelayo ${ }^{65}$, J. Peña-Rodriguez ${ }^{30}$, E.E. Pereira Martins ${ }^{39,8}$, J. Perez Armand ${ }^{21}$, C. Pérez Bertolli $^{8,41}$, M. Perlin ${ }^{8,41}$, L. Perrone ${ }^{56,48}$, S. Petrera ${ }^{45,46}$, T. Pierog ${ }^{41}$, M. Pimenta ${ }^{72}$, V. Pirronello ${ }^{58,47}$, M. Platino ${ }^{8}$, B. Pont $^{80}$, M. Pothast ${ }^{81,80}$, P. Privitera ${ }^{92}$, M. Prouza ${ }^{32}$, A. Puyleart ${ }^{89}$, S. Querchfeld ${ }^{38}$, J. Rautenberg ${ }^{38}$, D. Ravignani ${ }^{8}$, M. Reininghaus ${ }^{41,8}$, J. Ridky ${ }^{32}$, F. Riehn ${ }^{72}$, M. Risse ${ }^{44}$, V. Rizi ${ }^{57,46}$, W. Rodrigues de Carvalho ${ }^{21}$, J. Rodriguez Rojo ${ }^{11}$, M.J. Roncoroni ${ }^{8}$, S. Rossoni ${ }^{43}$, M. Roth ${ }^{41}$, E. Roulet ${ }^{1}$, A.C. Rovero ${ }^{5}$, P. Ruehl ${ }^{44}$, A. Saftoiu ${ }^{73}$, F. Salamida ${ }^{57,46}$, H. Salazar ${ }^{64}$, G. Salina ${ }^{51}$, J.D. Sanabria Gomez ${ }^{30}$, F. Sánchez ${ }^{8}$, E.M. Santos ${ }^{21}$, E. Santos ${ }^{32}$, F. Sarazin ${ }^{86}$, R. Sarmento ${ }^{72}$, C. Sarmiento-Cano ${ }^{8}$, R. Sato ${ }^{11}$, 
P. Savina ${ }^{56,48,34,94}$, C.M. Schäfer ${ }^{41}$, V. Scherini ${ }^{56,48}$, H. Schieler ${ }^{41}$, M. Schimassek ${ }^{39,8}$, M. Schimp ${ }^{38}$, F. Schlüter ${ }^{41,8}$, D. Schmidt ${ }^{39}$, O. Scholten ${ }^{84,15}$, P. Schovánek ${ }^{32}$, F.G. Schröder ${ }^{93,41}$, S. Schröder ${ }^{38}$, J. Schulte ${ }^{42}$, S.J. Sciutto ${ }^{4}$, M. Scornavacche ${ }^{8,41}$, A. Segreto ${ }^{53,47}$, S. Sehgal ${ }^{38}$, R.C. Shellard ${ }^{16}$, G. Sigl ${ }^{43}$, G. Silli ${ }^{8,41}$, O. Sima ${ }^{73, f}$, R. Šmída ${ }^{92}$, P. Sommers ${ }^{91}$, J.F. Soriano ${ }^{87}$, J. Souchard ${ }^{36}$, R. Squartini ${ }^{10}$, M. Stadelmaier ${ }^{41,8}$, D. Stanca ${ }^{73}$, S. Stanič ${ }^{76}$, J. Stasielak ${ }^{70}$, P. Stassi ${ }^{36}$, A. Streich ${ }^{39,8}$, M. Suárez-Durán ${ }^{14}$, T. Sudholz ${ }^{13}$, T. Suomijärvi ${ }^{37}$, A.D. Supanitsky ${ }^{8}$, Z. Szadkowski ${ }^{71}$, A. Tapia ${ }^{29}$, C. Taricco ${ }^{63,52}$, C. Timmermans ${ }^{81,80}$, O. Tkachenko ${ }^{41}$, P. Tobiska ${ }^{32}$, C.J. Todero Peixoto ${ }^{19}$, B. Tomé ${ }^{72}$, Z. Torrès ${ }^{36}$, A. Travaini ${ }^{10}$, P. Travnicek $^{32}$, C. Trimarelli ${ }^{57,46}$, M. Tueros ${ }^{4}$, R. Ulrich ${ }^{41}$, M. Unger ${ }^{41}$, L. Vaclavek ${ }^{33}$, M. Vacula ${ }^{33}$, J.F. Valdés Galicia ${ }^{68}$, L. Valore ${ }^{60,50}$, E. Varela ${ }^{64}$, A. Vásquez-Ramírez ${ }^{30}$, D. Veberič ${ }^{41}$, C. Ventura ${ }^{27}$, I.D. Vergara Quispe ${ }^{4}$, V. Verzi ${ }^{51}$, J. Vicha ${ }^{32}$, J. Vink $^{83}$, S. Vorobiov ${ }^{76}$, H. Wahlberg ${ }^{4}$, C. Watanabe ${ }^{26}$, A.A. Watson ${ }^{c}$, M. Weber ${ }^{40}$, A. Weindl ${ }^{41}$, L. Wiencke ${ }^{86}$, H. Wilczyński ${ }^{70}$, M. Wirtz ${ }^{42}$, D. Wittkowski ${ }^{38}$, B. Wundheiler ${ }^{8}$, A. Yushkov $^{32}$, O. Zapparrata ${ }^{14}$, E. Zas ${ }^{79}$, D. Zavrtanik ${ }^{76,77}$, M. Zavrtanik ${ }^{77,76}$, L. Zehrer ${ }^{76}$

${ }^{1}$ Centro Atómico Bariloche and Instituto Balseiro (CNEA-UNCuyo-CONICET), San Carlos de Bariloche, Argentina

${ }^{2}$ Centro de Investigaciones en Láseres y Aplicaciones, CITEDEF and CONICET, Villa Martelli, Argentina

${ }^{3}$ Departamento de Física and Departamento de Ciencias de la Atmósfera y los Océanos, FCEyN, Universidad de Buenos Aires and CONICET, Buenos Aires, Argentina

${ }^{4}$ IFLP, Universidad Nacional de La Plata and CONICET, La Plata, Argentina

${ }^{5}$ Instituto de Astronomía y Física del Espacio (IAFE, CONICET-UBA), Buenos Aires, Argentina

${ }^{6}$ Instituto de Física de Rosario (IFIR) - CONICET/U.N.R. and Facultad de Ciencias Bioquímicas y Farmacéuticas U.N.R., Rosario, Argentina

${ }^{7}$ Instituto de Tecnologías en Detección y Astropartículas (CNEA, CONICET, UNSAM), and Universidad Tecnológica Nacional - Facultad Regional Mendoza (CONICET/CNEA), Mendoza, Argentina

${ }^{8}$ Instituto de Tecnologías en Detección y Astropartículas (CNEA, CONICET, UNSAM), Buenos Aires, Argentina

${ }^{9}$ International Center of Advanced Studies and Instituto de Ciencias Físicas, ECyT-UNSAM and CONICET, Campus Miguelete - San Martín, Buenos Aires, Argentina

${ }^{10}$ Observatorio Pierre Auger, Malargüe, Argentina

${ }^{11}$ Observatorio Pierre Auger and Comisión Nacional de Energía Atómica, Malargüe, Argentina

12 Universidad Tecnológica Nacional - Facultad Regional Buenos Aires, Buenos Aires, Argentina

${ }^{13}$ University of Adelaide, Adelaide, S.A., Australia

14 Université Libre de Bruxelles (ULB), Brussels, Belgium

15 Vrije Universiteit Brussels, Brussels, Belgium

${ }^{16}$ Centro Brasileiro de Pesquisas Fisicas, Rio de Janeiro, RJ, Brazil

${ }^{17}$ Centro Federal de Educação Tecnológica Celso Suckow da Fonseca, Nova Friburgo, Brazil

${ }^{18}$ Instituto Federal de Educação, Ciência e Tecnologia do Rio de Janeiro (IFRJ), Brazil

${ }^{19}$ Universidade de São Paulo, Escola de Engenharia de Lorena, Lorena, SP, Brazil

${ }^{20}$ Universidade de São Paulo, Instituto de Física de São Carlos, São Carlos, SP, Brazil

${ }^{21}$ Universidade de São Paulo, Instituto de Física, São Paulo, SP, Brazil

${ }^{22}$ Universidade Estadual de Campinas, IFGW, Campinas, SP, Brazil

${ }^{23}$ Universidade Estadual de Feira de Santana, Feira de Santana, Brazil

${ }^{24}$ Universidade Federal do ABC, Santo André, SP, Brazil

${ }^{25}$ Universidade Federal do Paraná, Setor Palotina, Palotina, Brazil

${ }^{26}$ Universidade Federal do Rio de Janeiro, Instituto de Física, Rio de Janeiro, RJ, Brazil

${ }^{27}$ Universidade Federal do Rio de Janeiro (UFRJ), Observatório do Valongo, Rio de Janeiro, RJ, Brazil

${ }^{28}$ Universidade Federal Fluminense, EEIMVR, Volta Redonda, RJ, Brazil

${ }^{29}$ Universidad de Medellín, Medellín, Colombia

${ }^{30}$ Universidad Industrial de Santander, Bucaramanga, Colombia

${ }^{31}$ Charles University, Faculty of Mathematics and Physics, Institute of Particle and Nuclear Physics, Prague, Czech Republic

32 Institute of Physics of the Czech Academy of Sciences, Prague, Czech Republic 
${ }^{33}$ Palacky University, RCPTM, Olomouc, Czech Republic

34 CNRS/IN2P3, IJCLab, Université Paris-Saclay, Orsay, France

${ }^{35}$ Laboratoire de Physique Nucléaire et de Hautes Energies (LPNHE), Sorbonne Université, Université de Paris, CNRSIN2P3, Paris, France

${ }^{36}$ Univ. Grenoble Alpes, CNRS, Grenoble Institute of Engineering Univ. Grenoble Alpes, LPSC-IN2P3, 38000 Grenoble, France

${ }^{37}$ Université Paris-Saclay, CNRS/IN2P3, IJCLab, Orsay, France

38 Bergische Universität Wuppertal, Department of Physics, Wuppertal, Germany

${ }^{39}$ Karlsruhe Institute of Technology (KIT), Institute for Experimental Particle Physics, Karlsruhe, Germany

${ }^{40}$ Karlsruhe Institute of Technology (KIT), Institut für Prozessdatenverarbeitung und Elektronik, Karlsruhe, Germany

${ }^{41}$ Karlsruhe Institute of Technology (KIT), Institute for Astroparticle Physics, Karlsruhe, Germany

${ }^{42}$ RWTH Aachen University, III. Physikalisches Institut A, Aachen, Germany

${ }^{43}$ Universität Hamburg, II. Institut für Theoretische Physik, Hamburg, Germany

${ }^{44}$ Universität Siegen, Department Physik - Experimentelle Teilchenphysik, Siegen, Germany

${ }^{45}$ Gran Sasso Science Institute, L'Aquila, Italy

46 INFN Laboratori Nazionali del Gran Sasso, Assergi (L'Aquila), Italy

${ }^{47}$ INFN, Sezione di Catania, Catania, Italy

${ }^{48}$ INFN, Sezione di Lecce, Lecce, Italy

${ }^{49}$ INFN, Sezione di Milano, Milano, Italy

${ }^{50}$ INFN, Sezione di Napoli, Napoli, Italy

51 INFN, Sezione di Roma "Tor Vergata", Roma, Italy

52 INFN, Sezione di Torino, Torino, Italy

53 Istituto di Astrofisica Spaziale e Fisica Cosmica di Palermo (INAF), Palermo, Italy

54 Osservatorio Astrofisico di Torino (INAF), Torino, Italy

55 Politecnico di Milano, Dipartimento di Scienze e Tecnologie Aerospaziali , Milano, Italy

56 Università del Salento, Dipartimento di Matematica e Fisica "E. De Giorgi”, Lecce, Italy

57 Università dell'Aquila, Dipartimento di Scienze Fisiche e Chimiche, L'Aquila, Italy

58 Università di Catania, Dipartimento di Fisica e Astronomia, Catania, Italy

${ }^{59}$ Università di Milano, Dipartimento di Fisica, Milano, Italy

${ }^{60}$ Università di Napoli "Federico II", Dipartimento di Fisica "Ettore Pancini”, Napoli, Italy

${ }^{61}$ Università di Palermo, Dipartimento di Fisica e Chimica "E. Segrè", Palermo, Italy

62 Università di Roma "Tor Vergata", Dipartimento di Fisica, Roma, Italy

${ }^{63}$ Università Torino, Dipartimento di Fisica, Torino, Italy

64 Benemérita Universidad Autónoma de Puebla, Puebla, México

${ }^{65}$ Unidad Profesional Interdisciplinaria en Ingeniería y Tecnologías Avanzadas del Instituto Politécnico Nacional (UPIITA-IPN), México, D.F., México

66 Universidad Autónoma de Chiapas, Tuxtla Gutiérrez, Chiapas, México

${ }^{67}$ Universidad Michoacana de San Nicolás de Hidalgo, Morelia, Michoacán, México

${ }^{68}$ Universidad Nacional Autónoma de México, México, D.F., México

${ }^{69}$ Universidad Nacional de San Agustin de Arequipa, Facultad de Ciencias Naturales y Formales, Arequipa, Peru

${ }^{70}$ Institute of Nuclear Physics PAN, Krakow, Poland

${ }^{71}$ University of Łódź, Faculty of High-Energy Astrophysics,Lódź, Poland

${ }^{72}$ Laboratório de Instrumentação e Física Experimental de Partículas - LIP and Instituto Superior Técnico - IST, Universidade de Lisboa - UL, Lisboa, Portugal

73 "Horia Hulubei” National Institute for Physics and Nuclear Engineering, Bucharest-Magurele, Romania

${ }^{74}$ Institute of Space Science, Bucharest-Magurele, Romania

75 University Politehnica of Bucharest, Bucharest, Romania

76 Center for Astrophysics and Cosmology (CAC), University of Nova Gorica, Nova Gorica, Slovenia

${ }^{77}$ Experimental Particle Physics Department, J. Stefan Institute, Ljubljana, Slovenia

78 Universidad de Granada and C.A.F.P.E., Granada, Spain

${ }^{79}$ Instituto Galego de Física de Altas Enerxías (IGFAE), Universidade de Santiago de Compostela, Santiago de Compostela, Spain 
${ }^{80}$ IMAPP, Radboud University Nijmegen, Nijmegen, The Netherlands

${ }^{81}$ Nationaal Instituut voor Kernfysica en Hoge Energie Fysica (NIKHEF), Science Park, Amsterdam, The Netherlands

82 Stichting Astronomisch Onderzoek in Nederland (ASTRON), Dwingeloo, The Netherlands

${ }^{83}$ Universiteit van Amsterdam, Faculty of Science, Amsterdam, The Netherlands

${ }^{84}$ University of Groningen, Kapteyn Astronomical Institute, Groningen, The Netherlands

85 Case Western Reserve University, Cleveland, OH, USA

86 Colorado School of Mines, Golden, CO, USA

${ }^{87}$ Department of Physics and Astronomy, Lehman College, City University of New York, Bronx, NY, USA

${ }^{88}$ Louisiana State University, Baton Rouge, LA, USA

${ }^{89}$ Michigan Technological University, Houghton, MI, USA

${ }^{90}$ New York University, New York, NY, USA

${ }^{91}$ Pennsylvania State University, University Park, PA, USA

92 University of Chicago, Enrico Fermi Institute, Chicago, IL, USA

93 University of Delaware, Department of Physics and Astronomy, Bartol Research Institute, Newark, DE, USA

${ }^{94}$ University of Wisconsin-Madison, Department of Physics and WIPAC, Madison, WI, USA

${ }^{a}$ Fermi National Accelerator Laboratory, Fermilab, Batavia, IL, USA

${ }^{b}$ Max-Planck-Institut für Radioastronomie, Bonn, Germany

${ }^{c}$ School of Physics and Astronomy, University of Leeds, Leeds, United Kingdom

${ }^{d}$ Colorado State University, Fort Collins, CO, USA

$e^{e}$ now at Hakubi Center for Advanced Research and Graduate School of Science, Kyoto University, Kyoto, Japan

$f$ also at University of Bucharest, Physics Department, Bucharest, Romania

\section{Acknowledgements}

This work was supported by the Czech Republic grants of MEYS CR LTT18004 and LM2018102. 